\title{
Untargeted GC-TOFMS Analysis Reveals Metabolomic Changes in Salvia miltiorrhiza Bunge Leaf and Root in Response to Long-Term Drought Stress
}

\author{
Jin Zhang ${ }^{1,2,3,+}$, Yuekai Su ${ }^{1,2,+}$, Jingyu Li ${ }^{1,2} \oplus$, Zhenli Ren ${ }^{4}$, Guoqing Tian ${ }^{4}$ and Jianhua Wang ${ }^{1,2, *}$ \\ 1 State Key Laboratory of Crop Biology, Tai'an 271000, China; zhjin0214@163.com (J.Z.); \\ suyuekai163@163.com (Y.S.); sdauljy@163.com (J.L.) \\ 2 College of Agronomy, Shandong Agricultural University, Tai'an 271000, China \\ 3 Taishan Academy of Forestry Sciences, Tai'an 271000, China \\ 4 Shandong Danhong Pharmaceutical Co., Ltd., Heze 274000, China; renzhenli851@163.com (Z.R.); \\ love_youyezi@126.com (G.T.) \\ * Correspondence: wangih@sdau.edu.cn \\ + Equal contribution.
}

check for updates

Citation: Zhang, J.; Su, Y.; Li, J.; Ren, Z.; Tian, G.; Wang, J. Untargeted GC-TOFMS Analysis Reveals

Metabolomic Changes in Salvia miltiorrhiza Bunge Leaf and Root in Response to Long-Term Drought Stress. Horticulturae 2021, 7, 175. https://doi.org/10.3390/

horticulturae7070175

Academic Editor: Mercè Llugany

Received: 31 May 2021

Accepted: 25 June 2021

Published: 2 July 2021

Publisher's Note: MDPI stays neutral with regard to jurisdictional claims in published maps and institutional affiliations.

Copyright: (c) 2021 by the authors. Licensee MDPI, Basel, Switzerland. This article is an open access article distributed under the terms and conditions of the Creative Commons Attribution (CC BY) license (https:/ / creativecommons.org/licenses/by/ $4.0 /)$.

\begin{abstract}
Salvia miltiorrhiza Bunge (Danshen) is an important traditional Chinese medicine herb. This study aimed to investigate the drought-responsive metabolic profiling in S. miltiorrhiza using gas chromatography time-of-flight mass spectrometry (GC-TOFMS) analysis. Fifty day-old S. miltiorrhiza seedlings were treated with two (moderate drought, MD) and four weeks (high drought, HD) of withholding water. The S. miltiorrhiza leaf and root samples were prepared for the GC-TOFMS analysis. Differential metabolites with substantial changes in content in S. miltiorrhiza leaf and root were identified using multivariate and univariate statistics. A total of 178 and 157 annotated metabolites were detected in S. miltiorrhiza leaf and root, respectively. Multivariate analysis showed that significantly discriminant metabolites in S. miltiorrhiza leaf by drought were associated with "galactose metabolism" and "citrate cycle". In addition, the significantly discriminant metabolites in S. miltiorrhiza root were associated with "starch and sucrose metabolism". Univariate statistics showed that the content of succinic acid, D-glucose, and oxoglutaric acid in S. miltiorrhiza leaf was increased by drought (fold change, FC > 1.5). Allose, D-xylose, melibiose, mannose, sorbitol, quinic acid, sinigrin, and taurine in S. miltiorrhiza root were decreased by drought $(\mathrm{FC}<0.67)$. There were different metabolic profiles between S. miltiorrhiza leaf and root. However, the influence of drought stress on the pharmacological value and accumulation of bioactive constituents in S. miltiorrhiza should be further investigated.
\end{abstract}

Keywords: Salvia miltiorrhiza Bunge; metabolic profiling; drought stress; bioactive constituents; gas chromatography time-of-flight mass spectrometry

\section{Introduction}

Salvia miltiorrhiza Bunge (called Danshen in Chinese) is a plant of the genus Salvia of the Labiatae family. Its dried root and rhizome are important traditional Chinese medicine (TCM) materials. The bioactive constituents in the root of S. miltiorrhiza, including tanshinones, salvianolic acid B, and polysaccharides, exhibit potent bioactive activity such as free radical scavenging, antioxidant capacity, the prevention and treatment of vascular diseases, antianxiety, and anti-inflammatory effects [1-4]. Therefore, S. miltiorrhiza is widely used as a herbal medicine for the treatment of cardiovascular diseases, including coronary heart disease, atherosclerosis, myocardial infarction, and ischemia [4,5]. In addition, the market demand for S. miltiorrhiza remains high and its planting area is expanding with the increasing demand [6].

Drought has a devastating effect on crop production and quality $[7,8]$. Areas affected by water shortage account for $\sim 52 \%$ of the land in China and the annual grain production 
of global grain has been reduced by more than $1.00 \times 10^{11} \mathrm{~kg}$ due to drought [9]. In addition, drought has become one of the most important environmental factors and a major constraint on grain production and quality in many parts of the world.

There is increasing evidence showing that drought significantly affected the accumulation of bioactive constituents and secondary metabolites in medicinal plants $[6,10,11]$. Mild drought promoted the synthesis and accumulation of tanshinone, cryptotanshinone, and salvianolic acid B in S. miltiorrhiza root $[6,10]$. However, the content of rosmarinic acid was decreased by drought stress in S. miltiorrhiza root [6]. Cao et al. showed that the "sweating" process changed the accumulation of metabolites, including lipids, lipid-like molecules, phenylpropanoids, and polyketides in S. miltiorrhiza [12]. In addition, there is evidence showing that bioactive constituents (including aurantio-obtusin, aloe-emodin, rhein, and chrysophanol) in the seeds of Cassia obtusifolia were increased by moderate drought (80-50\% deficit irrigation) [12]. The determination of the metabolic profiling of the plant in response to drought stress is of significance for investigating TCM herbal quality $[6,11,13]$. However, the metabolic responses in S. miltiorrhiza in response to drought stress have not been reported systematically.

This study aimed to investigate the metabolic response in S. miltiorrhiza leaf and root to long-term drought stress using the gas chromatography time-of-flight mass spectrometry (GC-TOFMS) system. The effect of drought stress on metabolites in S. miltiorrhiza was identified, and different metabolites before and after drought stress were compared. This study might provide a theoretical basis for the cultivation and quality control of S. miltiorrhiza.

\section{Materials and Methods}

\subsection{Plant Materials and Drought Treatment}

S. miltiorrhiza seeds were obtained from the experimental base of Shandong Agricultural University, Tai'an, China. The seeds were planted into plastic basins $(12 \mathrm{~cm} \times 10 \mathrm{~cm})$ filled with matrix soil (nutrient soil:vermiculite $=7: 3, v / v$ ) and were placed in a laboratory greenhouse with $300 \mathrm{~mol} \cdot \mathrm{m}^{-2} \cdot \mathrm{s}^{-1}$ of illumination intensity, a 16:8 h light: dark cycle, and a $65 \%$ relative humidity. Fifty day-old S. miltiorrhiza seedlings with consistent growth were selected and treated with two (moderate drought stress, MD) and four weeks (high drought stress, HD) of withholding water. Seedlings treated with regular watering were used as normal control. Each treatment was performed in triplicate with two plants of each replication (6 replicates $\times 3$ groups). At the end of the experiments, the leaf and root samples were collected, snapped in liquid nitrogen, and stored at $-80^{\circ} \mathrm{C}$.

\subsection{Sample Preparation}

Samples ( 6 replicates $\times 3$ groups $\times 2$ types) were ground into powder and sieved with 50 mesh $(300 \mu \mathrm{m})$. Eighty milligrams of powdery sample were transferred to $1.5 \mathrm{~mL}$ Eppendorf tubes supplemented with extract solvents: methanol, acetonitrile, and water $(2: 2: 1 \mathrm{v} / \mathrm{v} / \mathrm{v})$. The samples were then ground $(60 \mathrm{~Hz}$ for $2 \mathrm{~min})$, sonicated three times $(8 \mathrm{~min})$, and rested at $-20^{\circ} \mathrm{C}$ for $1 \mathrm{~h}$. After centrifugation $\left(14,000 \times \mathrm{g}\right.$ for $10 \mathrm{~min}$ at $\left.4{ }^{\circ} \mathrm{C}\right)$, the supernatants were collected, filtered through a $0.22 \mu \mathrm{m}$ microfilter, vacuum dried, and stored at $-80^{\circ} \mathrm{C}$. Then, all samples $(n=36)$ were reconstituted with $100 \%$ methanol, vacuum dried, oximated with methoxyamine hydrochloride in pyridine $(50 \mu \mathrm{L}, 20 \mathrm{mg} / \mathrm{mL}$, for $90 \mathrm{~min}$ at $30^{\circ} \mathrm{C}$ ), and silylated with N-Methyl-N-(trimethylsilyl) trifluoroacetamide ( $50 \mu \mathrm{L}$, for $30 \mathrm{~min}$ at $37^{\circ} \mathrm{C}$ ). Quality-control samples were prepared by mixing equivalent amounts of all samples.

\subsection{GC-TOFMS Analysis}

The metabolic profiling in S. miltiorrhiza leaf and root was detected using the GCTOFMS system with an Agilent 4890 gas chromatograph (Palo Alto, CA, USA). A DB-5MS capillary column (30 m $\times 250 \mu \mathrm{m}$ i.d., $0.25-\mu \mathrm{m}$ film thickness; J \& W Scientific, Folsom, CA, USA) was used for sample separation $(1 \mu \mathrm{L})$. Helium was used as the carrier gas at a constant flow rate of $1.0 \mathrm{~mL} / \mathrm{min}$. The oven temperature was $75^{\circ} \mathrm{C}$ and increased to $300{ }^{\circ} \mathrm{C}$ 
$\left(15^{\circ} \mathrm{C} / \mathrm{min}\right)$. Electron ionization energy was $70 \mathrm{eV}$ and the full scan was $50-1000 \mathrm{~m} / \mathrm{z}$. The source and injection temperatures were set at $230^{\circ} \mathrm{C}$ and $270{ }^{\circ} \mathrm{C}$, respectively.

\subsection{Data Preprocessing and Annotation}

The XploreMET software (Metabo-Profile, Shanghai, China) and Chromma TOF software (V. 4.51.6.0, Leco, CA, USA) were used to analyze and process the range/scale data generated by GC-TOFMS. Metabolites were annotated using the JiaLib ${ }^{\mathrm{TM}}$ comprehensive proprietary mammalian metabolite database. The Kyoto Encyclopedia of Genes and Genomes (KEGG) database was used to analyze the pathways that were associated with metabolites in S. miltiorrhiza. Finally, Z-Score heatmap clustering of the differential metabolites was performed.

\subsection{Multivariate and Univariate Statistical Analysis}

Multivariate statistical analyses, including principal component analysis (PCA) and partial least squares discrimination analysis (PLS-DA), were carried out to compare the different metabolites between different experimental groups using the normalized data matrix. The significantly discriminant metabolites in $S$. miltiorrhiza leaf and root by drought stress were identified using the PLS-DA model with the criteria of variable importance in projection (VIP) $\geq 1$ and correlation coefficient (Corr.Coeff) $p$ value $<0.05$. The identification of differential metabolites with substantial changes in contents in response to drought between groups was performed using the threshold of fold change (FC) $\geq 1.5$ (increase) or $\leq 0.67$ (decrease) and $p<0.05$. The differential metabolites common to S. miltiorrhiza leaf and root were identified using the Venn diagram. The univariate statistical method Kruskal-Wallis H test was used to analyze the different levels of metabolites across three groups. A statistically significant difference was defined when $p$ value $<0.05$.

\section{Results}

\subsection{Metabolite Profiles of S. miltiorrhiza Leaf and Root Identified by GC-TOFMS Technology}

GC-TOFMS-based untargeted analysis produced a total of 447 and 349 metabolites in S. miltiorrhiza leaf and root, respectively, of which 178 (40\%) and 157 (45\%) metabolites were annotated (Supplementary Tables S1 and S2). The predominant metabolites in S. miltiorrhiza leaf and root were grouped into three metabolism categories: organic acids (22\% and $27 \%$ ), amino acids (24\% and $22 \%$ ), and carbohydrates (25\% and $24 \%$; Figure $1 \mathrm{~A}, \mathrm{~B})$. The results of the PCA analysis showed that there was a clear separation of S. miltiorrhiza leaf samples across groups (Figure S1A) and a high heterogeneity of S. miltiorrhiza root samples within groups (Figure S1B). However, the PLS-DA analysis showed that both S. miltiorrhiza leaf and root samples were separated clearly (Figure 1C,D). Accordingly, we used all the samples $(n=36)$ to analyze the metabolic profiles in S. miltiorrhiza leaf and root.

\subsection{Identification of Differential Metabolites in S. miltiorrhiza Leaf and Root}

Based on the PLS-DA model analysis and the criteria of VIP $\geq 1$ and $p$ value $<0.05$, we identified that MD and HD treatment induced 63 and 71 significantly discriminant metabolites in S. miltiorrhiza leaf compared with control, respectively (Supplementary Table S3). In addition, MD and HD treatment induced 39 and 44 significantly discriminant metabolites in S. miltiorrhiza root compared with control, respectively (Supplementary Table S3).

Most of the above metabolites with substantial increase (FC $\geq 1.5$ and $p<0.05)$ or decrease (FC $\leq 0.67$ and $p<0.05)$, including 62 and 45 metabolites in S. miltiorrhiza leaf, were induced by HD and MD treatments ( 25 common metabolites, six downregulated and 19 upregulated; Figure 2A) as well as 34 and 44 metabolites in S. miltiorrhiza root induced by HD and MD treatments, respectively (14 common metabolites, 12 downregulated and two upregulated; Figure 2B). The common metabolites are listed in Table 1. 

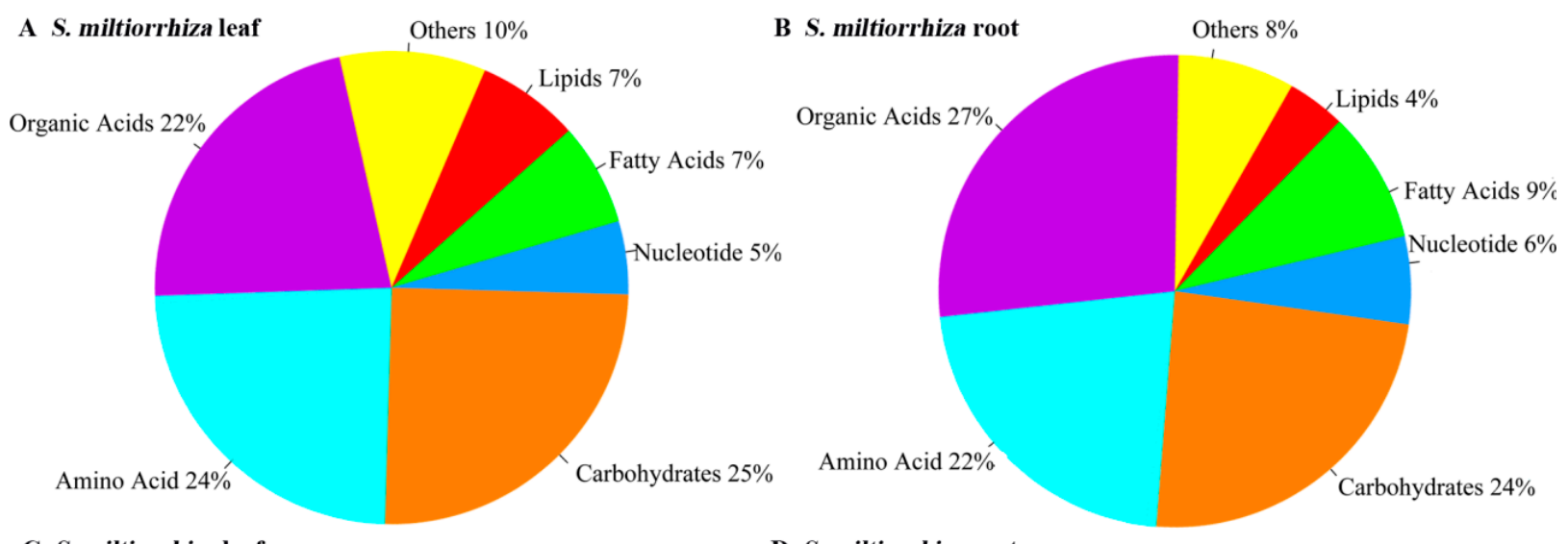

C S. miltiorrhiza leaf

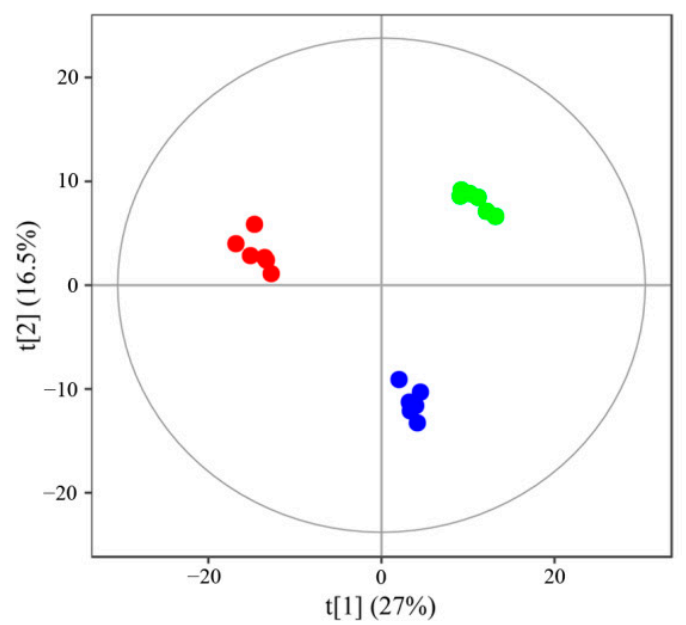

D S. miltiorrhiza root

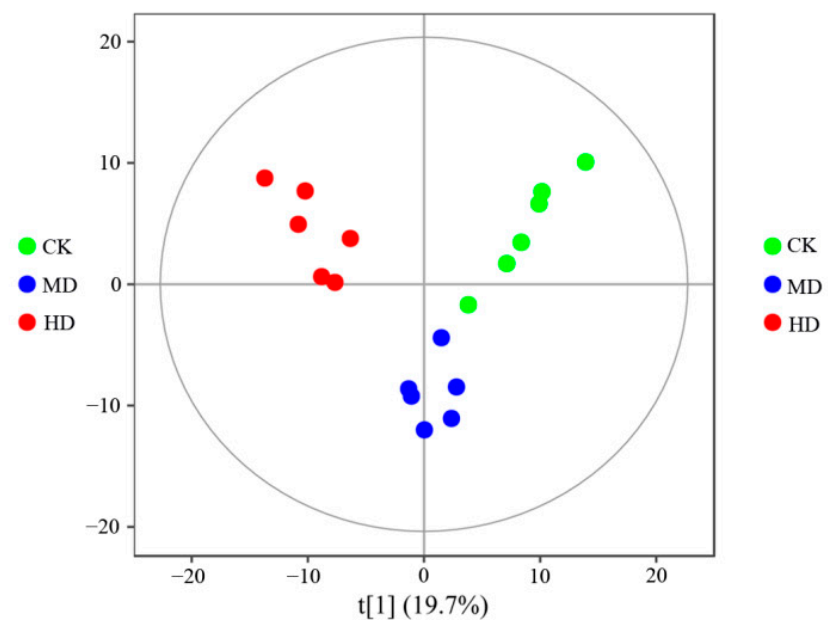

Figure 1. Overview of the metabolites in Salvia miltiorrhiza Bunge leaf and root. (A,B): The pie chart indicating the distribution of annotated metabolites. (C,D): The results of the partial least squares discrimination analysis (PLS-DA) of analyzed samples. MD: moderate drought. HD: high (severe) drought. CK: control.

\section{A S. miltiorrhiza leaf} $\mathrm{MD}$ versus $\mathrm{CK}$

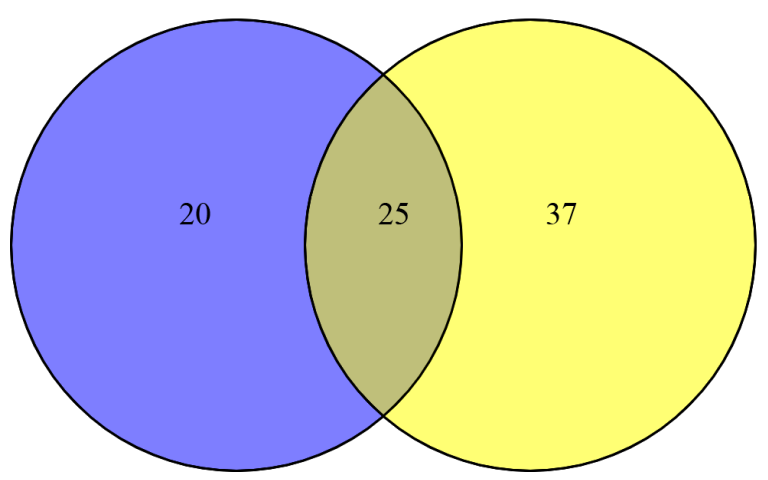

B S. miltiorrhiza root $\mathrm{MD}$ versus $\mathrm{CK}$ HD versus $\mathrm{CK}$

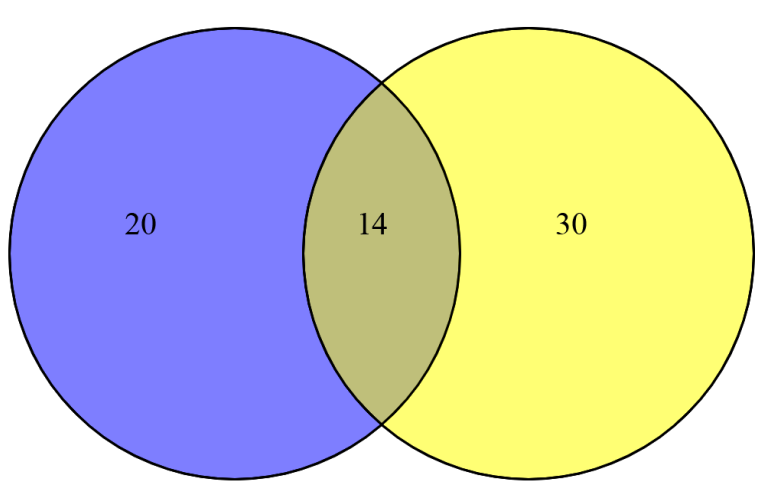

Figure 2. The Venn diagram of differential metabolites in Salvia miltiorrhiza Bunge. (A,B): the Venn diagram indicating differential metabolites in S. miltiorrhiza leaf and root, respectively. Differential metabolites were identified using the thresholds of fold change $(\mathrm{FC}) \geq 1.5$ or $\leq 0.67(|\log 2 \mathrm{FC}| \geq 0.585)$ and $p<0.05$. 
Table 1. Significantly differential metabolites in Salvia miltiorrhiza Bunge leaf and root induced by drought stress by univariate statistics.

\begin{tabular}{|c|c|c|c|c|c|c|c|}
\hline \multirow{2}{*}{ Class } & \multirow{2}{*}{ Name } & \multirow{2}{*}{ HMDBID } & \multirow{2}{*}{ KeggID } & \multicolumn{2}{|c|}{ HD versus $C K$} & \multicolumn{2}{|c|}{ MD versus $C K$} \\
\hline & & & & $p$ Value & FC & $p$ Value & FC \\
\hline \multicolumn{8}{|c|}{ S. miltiorrhiza leaf } \\
\hline \multirow{6}{*}{ Amino acids } & Ratio of L-Glutamic acid & HMDB00148 & $\mathrm{C} 00025$ & & & & \\
\hline & to Oxoglutaric acid & HMDB00208 & C00026 & $5.50 \mathrm{e}-03$ & 0.06 & $2.40 \mathrm{e}-02$ & 0.35 \\
\hline & L-Lysine & HMDB00182 & C00047 & $3.50 \mathrm{e}-03$ & 2.58 & $4.50 \mathrm{e}-04$ & 1.85 \\
\hline & L-Leucine & HMDB00687 & C00123 & $5.00 \mathrm{e}-06$ & 0.11 & $2.00 \mathrm{e}-04$ & 0.40 \\
\hline & L-Histidine & HMDB00177 & C00135 & $6.10 \mathrm{e}-03$ & 4.47 & $6.50 \mathrm{e}-06$ & 2.98 \\
\hline & L-Alloisoleucine & HMDB00557 & NA & $1.60 \mathrm{e}-02$ & 21.98 & $2.50 \mathrm{e}-05$ & 41.37 \\
\hline \multirow[t]{7}{*}{ Carbohydrates } & Sucrose & HMDB00258 & C00089 & $2.70 \mathrm{e}-02$ & 2.48 & $1.20 \mathrm{e}-04$ & 2.46 \\
\hline & Sorbitol & HMDB00247 & C00794 & $4.90 \mathrm{e}-02$ & 0.11 & $4.20 \mathrm{e}-02$ & 0.07 \\
\hline & Mannitol & HMDB00765 & C00392 & $3.30 \mathrm{e}-02$ & 1.94 & $1.60 \mathrm{e}-02$ & 1.53 \\
\hline & Lactulose & HMDB00740 & C07064 & $2.10 \mathrm{e}-02$ & 3.90 & $2.30 \mathrm{e}-05$ & 3.05 \\
\hline & D-Xylose & HMDB00098 & C00181 & $5.80 \mathrm{e}-03$ & 1.95 & $8.00 \mathrm{e}-03$ & 1.78 \\
\hline & D-Glucose & HMDB00122 & C00031 & $2.40 \mathrm{e}-02$ & 1.81 & $1.40 \mathrm{e}-02$ & 1.71 \\
\hline & beta-Lactose & HMDB41627 & C01970 & $5.30 \mathrm{e}-03$ & 2.13 & $1.30 \mathrm{e}-04$ & 6.38 \\
\hline Fatty acids & Docosahexaenoic acid & HMDB02183 & C06429 & $2.70 \mathrm{e}-03$ & 0.16 & $2.80 \mathrm{e}-02$ & 0.49 \\
\hline Indoles & Indoleacetic acid & HMDB00197 & C00954 & $4.90 \mathrm{e}-02$ & 0.39 & $3.60 \mathrm{e}-02$ & 2.48 \\
\hline Lipids & MG182 & HMDB11568 & NA & $1.70 \mathrm{e}-02$ & 3.01 & $3.20 \mathrm{e}-03$ & 1.94 \\
\hline \multirow[t]{4}{*}{ Nucleotide } & Ratio of Uridine & HMDB00296 & C00299 & $1.30 \mathrm{e}-05$ & 0.50 & $3.00 \mathrm{e}-06$ & 0.24 \\
\hline & to Cytidine & HMDB00089 & C00475 & $1.30 \mathrm{e}-05$ & 0.50 & $3.00 \mathrm{e}-06$ & 0.24 \\
\hline & Ratio of Uracil & HMDB00300 & C00106 & & & $1.20 \mathrm{e}-02$ & 2.52 \\
\hline & to Uridine & HMDB00296 & C00299 & $2.70 \mathrm{e}-02$ & 1.76 & $1.20 \mathrm{e}-02$ & \\
\hline \multirow[t]{7}{*}{ Organic acids } & trans-Ferulic acid & HMDB00954 & C01494 & $5.50 \mathrm{e}-05$ & 1.65 & $5.80 \mathrm{e}-04$ & 1.51 \\
\hline & Succinic acid & HMDB00254 & C00042 & $3.20 \mathrm{e}-07$ & 3.71 & $6.60 \mathrm{e}-03$ & 1.84 \\
\hline & Maleic acid & HMDB00176 & C01384 & $5.00 \mathrm{e}-02$ & 3.82 & $6.80 \mathrm{e}-05$ & 1.78 \\
\hline & L-Malic acid & HMDB00156 & C00149 & $5.60 \mathrm{e}-03$ & 3.14 & $1.00 \mathrm{e}-02$ & 2.90 \\
\hline & Fumaric acid & HMDB00134 & C00122 & $3.70 \mathrm{e}-02$ & 1.37 & $2.30 \mathrm{e}-05$ & 1.59 \\
\hline & 4-Hydroxycinnamic acid & HMDB02035 & C00811 & $2.90 \mathrm{e}-02$ & 2.02 & $4.50 \mathrm{e}-06$ & 1.69 \\
\hline & 3-Aminosalicylic acid & HMDB01972 & NA & $3.50 \mathrm{e}-04$ & 0.08 & $2.80 \mathrm{e}-04$ & 0.04 \\
\hline Vitamin & Pantothenic acid & HMDB00210 & C00864 & $3.10 \mathrm{e}-02$ & 2.46 & $3.90 \mathrm{e}-04$ & 1.77 \\
\hline \multicolumn{8}{|c|}{ S. miltiorrhiza root } \\
\hline \multirow[t]{2}{*}{ Amino acid } & Urea & HMDB00294 & C00086 & $1.50 \mathrm{e}-04$ & 0.16 & $1.60 \mathrm{e}-04$ & 0.20 \\
\hline & L-Alloisoleucine & HMDB00557 & NA & $1.00 \mathrm{e}-03$ & 20.07 & $1.60 \mathrm{e}-03$ & 59.69 \\
\hline \multirow[t]{9}{*}{ Carbohydrates } & Sorbitol & HMDB00247 & C00794 & $1.30 \mathrm{e}-03$ & 0.23 & $2.90 \mathrm{e}-02$ & 0.50 \\
\hline & Ribonolactone & HMDB01900 & $\mathrm{C} 02674$ & $6.00 \mathrm{e}-04$ & 0.59 & $1.60 \mathrm{e}-04$ & 0.65 \\
\hline & Ratio of L-Arabinose & HMDB00646 & C00259 & & & & \\
\hline & to L-Arabitol & HMDB01851 & C00532 & $1.70 \mathrm{e}-02$ & 0.11 & 4.70 & 0.30 \\
\hline & Melibiose & HMDB00048 & C05402 & $1.60 \mathrm{e}-02$ & 0.42 & $6.50 \mathrm{e}-03$ & 0.29 \\
\hline & Isomaltose & HMDB02923 & C00252 & $1.90 \mathrm{e}-02$ & 5.43 & $1.50 \mathrm{e}-02$ & 5.26 \\
\hline & Glucose 6-phosphate & HMDB01401 & C00092 & $1.10 \mathrm{e}-02$ & 0.47 & $2.80 \mathrm{e}-02$ & 0.63 \\
\hline & Glucose 1-phosphate & HMDB01586 & C00103 & $5.70 \mathrm{e}-03$ & 0.53 & $7.20 \mathrm{e}-04$ & 0.60 \\
\hline & D-Mannose & HMDB00169 & C00159 & $6.70 \mathrm{e}-03$ & 0.53 & $1.00 \mathrm{e}-02$ & 0.61 \\
\hline Fatty acids & Myristoleic acid & HMDB02000 & C08322 & $4.10 \mathrm{e}-02$ & 0.56 & $2.30 \mathrm{e}-02$ & 0.54 \\
\hline Lipids & MG182 & HMDB11568 & NA & $5.80 \mathrm{e}-03$ & 0.55 & $1.00 \mathrm{e}-03$ & 0.30 \\
\hline Nucleotide & Uridine & HMDB00296 & C00299 & $2.80 \mathrm{e}-02$ & 0.52 & $4.50 \mathrm{e}-02$ & 0.55 \\
\hline Organic acids & Taurine & HMDB00251 & $\mathrm{C} 00245$ & $2.60 \mathrm{e}-02$ & 0.42 & $4.40 \mathrm{e}-03$ & 0.19 \\
\hline
\end{tabular}

VIP: variable importance in projection; Corr.Coeff: correlation coefficient; FC: fold change; KEGG: Kyoto Encyclopedia of Genes and Genomes. NA: not applicable.

\subsection{KEGG Pathway Annotation}

The KEGG pathways related to the metabolites with substantial increase and decrease in S. miltiorrhiza leaf and root are shown in Figure 3A,B. The metabolites in S. miltiorrhiza leaf were related to "valine, leucine, and isoleucine" (hsa00290, including downregulated L-Leucine), "galactose metabolism" (hsa00052, including downregulated sorbitol and upregulated sucrose and D-glucose), and "citrate cycle (TCA cycle)" (hsa00020, including upregulated succinic acid and fumaric acid; Figure 3A), and the differential metabolites 
induced by HD treatment in S. miltiorrhiza leaf were related to "butanoate metabolism" (hsa00650, including upregulated succinic acid), "D-glutamine and D-glutamate metabolism" (hsa00471, including upregulated oxoglutaric acid), and "arginine biosynthesis" (hsa00220, including upregulated fumaric acid and oxoglutaric acid).
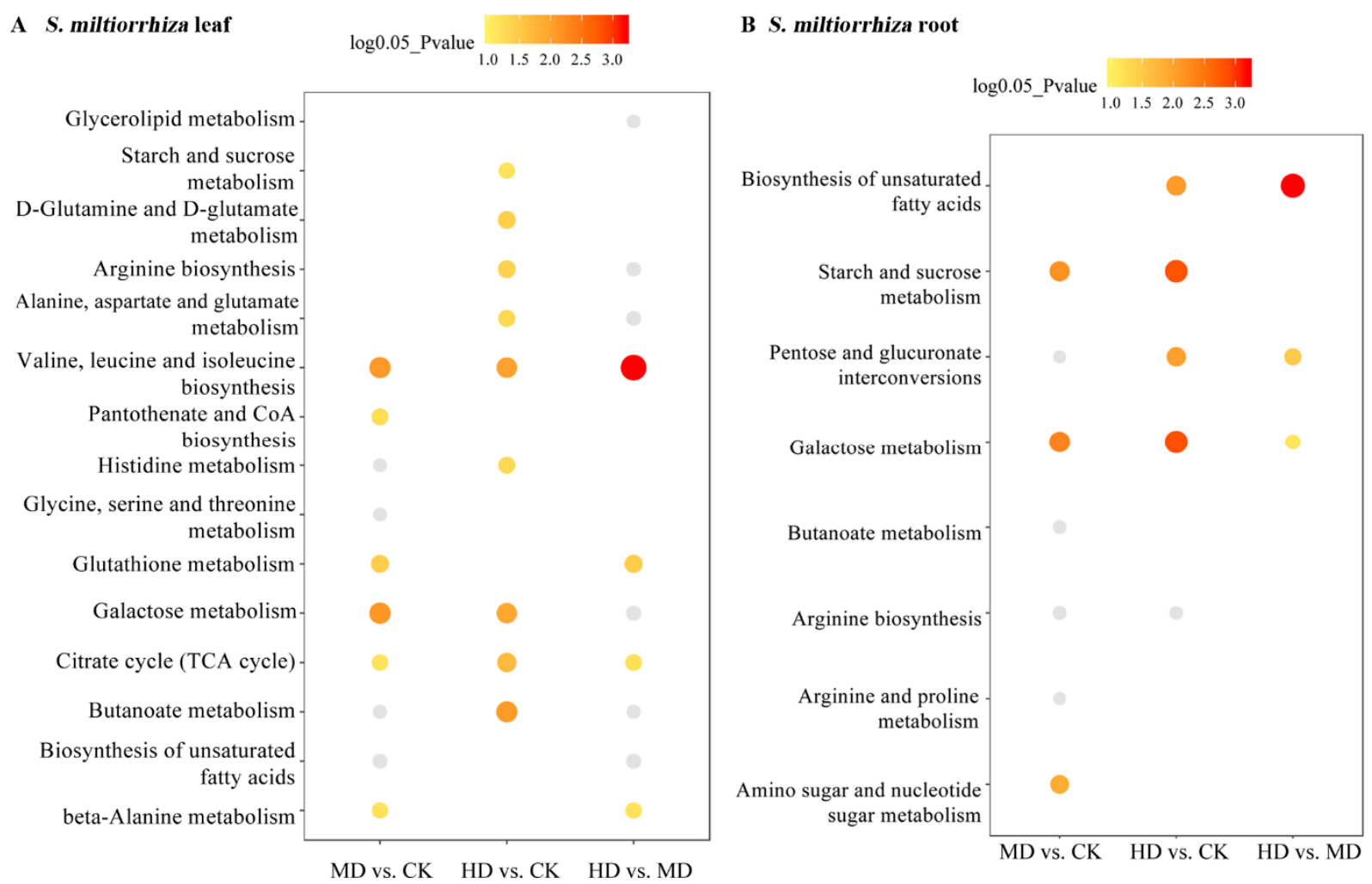

Figure 3. The metabolic pathway enrichment analysis of differential metabolites in Salvia miltiorrhiza Bunge. (A,B): the pathways related to differential metabolites in S. miltiorrhiza leaf and root, respectively. Significantly differential metabolites were identified using the thresholds of fold change $(\mathrm{FC}) \geq 1.5$ or $\leq 0.67(\mid \log 2 \mathrm{FCl} \geq 0.585)$ and $p<0.05$.

In addition, we demonstrated that metabolites with substantial changes in S. miltiorrhiza root were related to "starch and sucrose metabolism" (hsa00500, including upregulated D-maltose and isomaltose) and hsa00052 (including downregulated glucose 1-phosphate, melibiose, and sorbitol; Figure 3B), and the HD-induced metabolites with substantial changes in S. miltiorrhiza root were associated with "pentose and glucuronate interconversions" (hsa00040, including downregulated glucose 1-phosphate). The sample clustering of metabolites enriched with the above pathways in S. miltiorrhiza leaf and root is shown in Figure S2A,B.

\subsection{Metabolic Profiles of Metabolites Related to the Biosynthesis of Terpenoids in S. miltiorrhiza}

The major pathways and the levels of key metabolites related to the biosynthesis of terpenoids are shown in Figure 4. The glycolysis and TCA cycle metabolites are the primary substances of terpenoid biosynthesis: the mevalonic acid (MVA) and 2-methyl-D-erythritol 4-phosphate (methylerythritol phosphate, MEP) pathways (Figure 4A). We showed that drought stress-induced differential metabolites were related to glycolysis and TCA cycle in S. miltiorrhiza leaf (Figure 4B). L-Pipecolic acid, phosphoenolpyruvic acid, isomaltose, and tartaric acid were the metabolites with the highest contents in S. miltiorrhiza leaf (peak area $>40,000$, data not shown). The MD and HD stress increased the contents of succinic acid (1.84 and 3.71 FC), fumaric acid (1.59 and 1.37 FC), D-glucose (1.71 and 1.81 FC), oxoglutaric acid (MD: 10.83 FC), and L-malic acid (2.90 and 3.14 FC) compared with control (Figure 4B), while the HD stress decreased the accumulation of glucose 6-phosphate 
(0.43 FC), glucose 6-phosphate/D-glucose ratio (0.26 FC), and fumaric acid/L-malic acid ratio ( 0.39 FC; $p<0.05$ by Kruskal-Wallis $\mathrm{H}$ test).

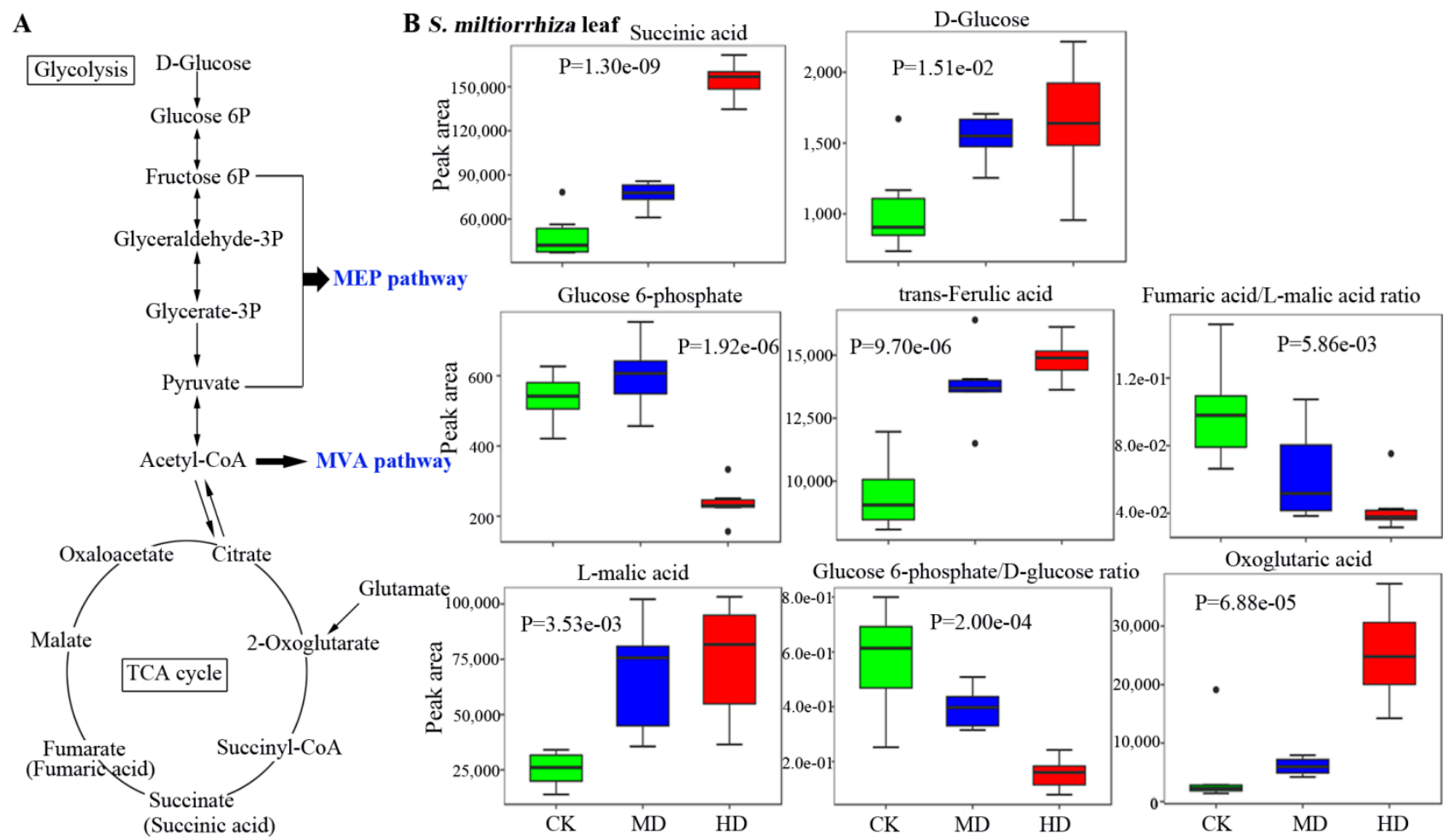

Figure 4. Metabolic profiles of terpenoid biosynthesis metabolites in Salvia miltiorrhiza Bunge. (A): the metabolic pathways (glycolysis and TCA cycle) producing primary substances of terpenoid biosynthesis. MVA, mevalonic acid. MEP, 2-methylD-erythritol 4-phosphate/methylerythritol phosphate. (B): the profiles of differential metabolites in S. miltiorrhiza leaf. CK, control. MD, moderate drought. HD, high (severe) drought. Univariate statistic method Kruskal-Wallis $\mathrm{H}$ test was used to analyze the different levels of metabolites. Data were expressed as median and range (interquartile range).

Also, we identified that metabolites with the highest contents in S. miltiorrhiza root were fructose 6-phosphate, galactinol, allose, and sinigrin (peak area $>10,000$, data not shown). We also identified that three differential metabolites (glucose 6-phosphate, glucose 1-phosphate, and D-glucose) that were related to glycolysis and TCA cycle were decreased in S. miltiorrhiza root by drought stress, especially by HD treatment (Figure 5). Besides, MD and HD stresses gradually decreased the contents of allose ( 0.87 and $0.61 \mathrm{FC})$, D-xylose (0.55 and $0.16 \mathrm{FC})$, melibiose ( 0.29 and $0.42 \mathrm{FC})$, and D-mannose ( 0.61 and $0.53 \mathrm{FC}$ ) compared with control (Figure 5), while the content of isomaltose was increased by drought (MD and HD: 5.26 and 5.43 FC; $p<0.05$ by Kruskal-Wallis $H$ test). These results might show that the biosynthesis of terpenoids and the accumulation of carbohydrate metabolites in $S$. miltiorrhiza root were inhibited by drought stress.

\subsection{Drought Decreases the Bioactive Constituents in S. miltiorrhiza Root}

We also showed that the contents of bioactive constituents in S. miltiorrhiza root, including beta-sitosterol ( 0.26 and $0.47 \mathrm{FC})$, sorbitol ( 0.50 and $0.23 \mathrm{FC})$, myristoleic acid ( 0.54 and $0.56 \mathrm{FC})$, quinic acid ( 0.85 and $0.37 \mathrm{FC}$ ), sinigrin ( 0.67 and $0.47 \mathrm{FC})$, and taurine (0.19 and $0.42 \mathrm{FC}$ ), were decreased by drought stress compared with control (Figure 6). However, the content of L-alloisoleucine in S. miltiorrhiza root was increased by drought (MD and HD: 59.69 and 20.07 FC). These results showed that the pharmacological value of S. miltiorrhiza might be decreased by drought stress. 


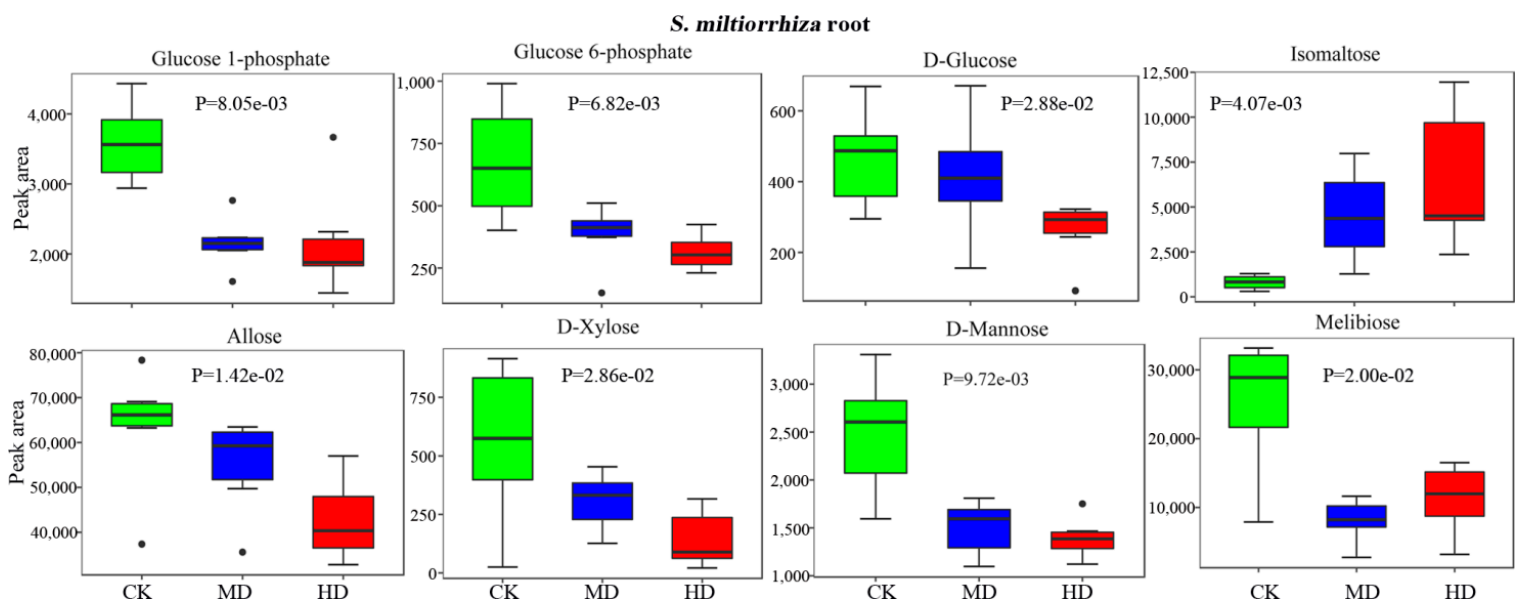

Figure 5. Metabolic profiles of glycolysis metabolites and carbohydrate metabolites in Salvia miltiorrhiza Bunge root. CK: control. MD: moderate drought. HD: high (severe) drought. Univariate statistic method Kruskal-Wallis $\mathrm{H}$ test was used to analyze the different levels of metabolites. Data were expressed as median and range (interquartile range).

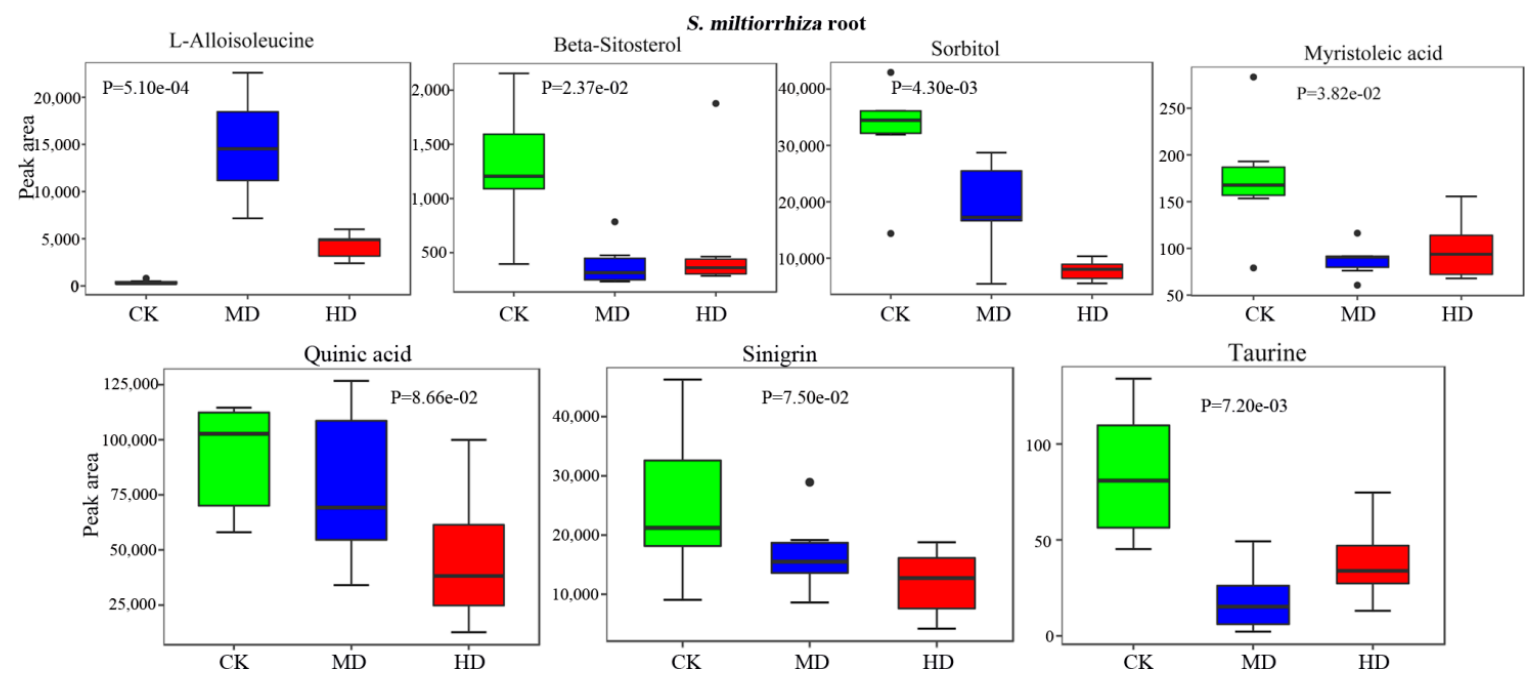

Figure 6. Metabolic profiles of several bioactive constituents in Salvia miltiorrhiza Bunge root. CK: control. MD: moderate drought. HD: high (severe) drought. Univariate statistic method Kruskal-Wallis H test was used to analyze the different levels of metabolites. Data were expressed as median and range (interquartile range).

\section{Discussion}

Both biotic and abiotic stresses influence the quality, production, and accumulation of secondary metabolites in the plant $[8,13,14]$. Our present study showed that most of the differential metabolites in S. miltiorrhiza leaf were increased by drought stress and most differential metabolites in S. miltiorrhiza root were decreased by drought stress. Moreover, we showed that most of the metabolites related to the biosynthesis of terpenoids, glycolysis, and TCA cycle in S. miltiorrhiza leaves were upregulated by drought stress. However, several bioactive constituents and most of the metabolites related to the biosynthesis of terpenoids, glycolysis, TCA cycle, and carbohydrate metabolism in S. miltiorrhiza root were decreased by drought stress. These results showed that the pharmacological value and the accumulation of bioactive constituents in S. miltiorrhiza leaf and root were influenced by drought.

Plant growth and development are frequently stressed by various environmental factors (including salinity, drought, temperature, and water), organisms (diseases, pests, and weeds), and deficiency of nutrients (including nitrogen, phosphorus, and potassium) [14-17]. The above factors influence transcriptomics, proteomics, and metabolomics 
in plants [17-19]. Chai et al. [18] showed that most of the metabolites in Vitis amurensis plantlets identified by the GC-TOMFS system were grouped into three categories (carbohydrates, amino acids, and organic acids) and were positively correlated with decreasing temperatures. They showed that metabolites including galactinol, melibiose, xylose, glucose, maltose, lactose, psicose, raffinose, fructose, mannose, glycerol, 2-oxoglutarate, glycine, alanine, arginine, glutamine, aspartate, succinate, malate, and fumarate were gradually increased by cold treatment at $4{ }^{\circ} \mathrm{C}$ for $24 \mathrm{~h}$ and $72 \mathrm{~h}$ [18]. Zhu et al. [10] revealed that the accumulation of saikosaponin $\mathrm{a} / \mathrm{b}$ in Bupleurum chinense DC. seedlings were gradually increased with the degree of drought stress. They demonstrated that the contents of saikosaponin $\mathrm{a} / \mathrm{b}$ in B. chinense DC. seedlings were increased by mild drought stress (water deficit for 3 and 6 days) and the content of saikosaponin a was decreased by severe drought stress (water deficit for 9 days) compared with control. Chen et al. [20] demonstrated that the amounts of guanosine and succinic acid in Pinellia ternata were reduced by drought stress compared with control treatment. In addition, Jia et al. [11] identified that the contents of calycosin-7-O- $\beta$-D-glycoside and ononin in Radix Astragali roots were enhanced with the degree of drought stress. These results showed that drought stress has different influences on the biosynthesis and accumulation of metabolites.

Our present study showed that drought stress (two and four weeks of withholding water) reduced the contents of carbohydrate metabolites in S. miltiorrhiza root, including glucose 1-phosphate, glucose 6-phosphate, D-glucose, allose, D-xylose, D-mannose, and melibiose. However, we also showed that the contents of oxoglutaric acid, trans-ferulic acid, D-glucose, and succinic acid in S. miltiorrhiza leaf were increased by drought stress. Moreover, the contents of bioactive constituents in S. miltiorrhiza root, including beta-sitosterol, sorbitol, myristoleic acid, quinic acid, sinigrin, and taurine, were gradually decreased by moderate and severe drought stresses. These results indicated different metabolic profiling between S. miltiorrhiza root and leaf in response to drought stress. Moreover, drought might be a major constraint on the accumulation of bioactive constituents and the pharmacological value of $S$. miltiorrhiza root.

Sugars mainly come from photosynthesis, gluconeogenesis, and polysaccharide degradation in plant cells. In this study, the contents of D-glucose, allose, D-xylose, D-mannose, melibiose, beta-sitosterol, sorbitol, myristoleic acid, quinic acid, sinigrin, and taurine in $S$. miltiorrhiza root increased significantly under drought stress. Quinic acid has been shown to exhibit potent bioactive activity, including anti-neuroinflammatory, antivirulence, radioprotection, and anti-oxidant capacity [21-23]. Sinigrin has anti-inflammatory, anti-asthmatic, and anti-atherosclerosis activities [24-26]. Lee et al. [25] showed that sinigrin reduced the lipopolysaccharide-induced production of inflammatory cytokine tumor necrosis factor (TNF)- $\alpha$ and interleukin (IL)-6 in RAW 264.7 macrophages and inhibited the nuclear factorkappa B (NF-kB)/MAPK pathway. Quinic acid and sinigrin both inhibit TNF- $\alpha$-induced expression of vascular cell adhesion molecule-1 (VCAM-1) [21,26]. In addition, taurine shows a neuroprotection function in a mouse model with the manganism-induced locomotor deficit [27]. The above evidence shows that long-term drought stress reduced the accumulation of the above bioactive constituents and might decrease the pharmacological value of the $S$. miltiorrhiza root.

Salvianolic acid B and tanshinones (including tanshinone I/IIA and cryptotanshinone) are diterpenoids: secondary metabolites of S. miltiorrhiza [28]. Photosynthesis, glycolysis, and the TCA cycle provide basic stacking modules for the biosynthesis of terpenoids through the MVA and MEP pathways [28]. Tanshinones have antitumor properties [29-31]. Studies show that drought stress reduced the terpenoid emissions of cork oak [32] and the needle terpenoid contents of the coastal-provenance Douglas fir [33]. The decreased glycolysis and the TCA cycle in S. miltiorrhiza root might show that the biosynthesis of terpenoids is inhibited by drought stress. However, Liu et al. showed that the amounts of salvianolic acid B and tanshinones in S. miltiorrhiza root were decreased and increased by drought stress, respectively [6]. In addition, Xue et al. showed that drought stress (80-50\% deficit irrigation) increased the contents of aurantio-obtusin, aloe-emodin, rhein, 
and chrysophanol in C. obtusifolia seeds [12]. However, our study did not identify terpene metabolites in S. miltiorrhiza leaf and root using GC-TOFMS-based untargeted metabolic analysis. The studies from Liu et al. [6] and Xue et al. [12] showed that drought stress might be a factor conducive to the accumulation of tanshinones in S. miltiorrhiza. The results showed that drought stress is a controversial factor in the accumulation of bioactive constituents and the pharmacological value of S. miltiorrhiza.

\section{Conclusions}

In summary, this study demonstrated that the long-term drought stress increased the glycolysis and TCA cycle in S. miltiorrhiza leaf. In addition, drought stress decreased the glycolysis and TCA cycle metabolism and the biosynthesis of the bioactive constituents in S. miltiorrhiza root, including D-glucose, allose, D-xylose, D-mannose, melibiose, betasitosterol, sorbitol, myristoleic acid, quinic acid, sinigrin, and taurine. These results demonstrated that there were different metabolic profiles between the leaf and root of S. miltiorrhiza. However, the influence of drought stress on the pharmacological value and accumulation of bioactive constituents in S. miltiorrhiza should be further investigated by more experiments.

Supplementary Materials: The following are available online at https://www.mdpi.com/article/10 .3390/horticulturae7070175/s1, Table S1. All metabolites in Salvia miltiorrhiza Bunge leaf. Table S2. All metabolites in Salvia miltiorrhiza Bunge root. Table S3. Significantly discriminant and differential metabolites in Salvia miltiorrhiza Bunge leaf and root induced by drought stress. Figure S1. Principal component analysis (PCA) of the Salvia miltiorrhiza Bunge leaf and root samples. MD, moderate drought. HD, high (severe) drought. CK, control.

Author Contributions: Conceptualization: J.Z. and J.W.; methodology: J.Z. and J.L.; software and formal analysis: J.Z., Y.S. and J.L.; investigation: Y.S. and J.Z.; resources and data curation: J.Z., J.L., Z.R. and G.T.; writing—original draft preparation: J.Z.; writing—review and editing: Y.S. and J.W.; funding acquisition: J.W., Z.R. and G.T.; supervision: J.W.; project administration: J.Z. All authors have read and agreed to the published version of the manuscript.

Funding: This work was supported by the Shandong Modern Agricultural Industry Technical System Project (SDAIT-20-04), the Key Research and Development Plan of Shandong Province (2017CXGC1302; 2019LYXZ021), and National Key R\&D Program of China (2017YFC1702705).

Institutional Review Board Statement: Not applicable.

Informed Consent Statement: Not applicable.

Data Availability Statement: The original metabolic data are available from the corresponding author (Wang J.: wangjh@sdau.edu.cn) with reasonable request.

Conflicts of Interest: The authors have declared no conflict of interest.

\section{Abbreviations}

$\begin{array}{ll}\text { FC } & \text { fold change; } \\ \text { GC-TOFMS } & \text { gas chromatography time-of-flight mass spectrometry; } \\ \text { HD } & \text { high drought; } \\ \text { KEGG } & \text { Kyoto Encyclopedia of Genes and Genomes; } \\ \text { MD } & \text { moderate drought; } \\ \text { MEP } & \text { 2-methyl-D-erythritol 4-phosphate; } \\ \text { MVA } & \text { mevalonic acid; } \\ \text { NF-kB } & \text { nuclear factor-kappa B; } \\ \text { PCA } & \text { principal component analysis; } \\ \text { PLS-DA } & \text { partial least squares discrimination analysis; } \\ \text { TCA } & \text { citrate cycle; } \\ \text { TCM } & \text { traditional Chinese medicine; } \\ \text { VCAM-1 } & \text { vascular cell adhesion molecule-1; } \\ \text { VIP } & \text { variable importance in projection; }\end{array}$




\section{References}

1. Lin, Y.-S.; Peng, W.-H.; Shih, M.-F.; Cherng, J.-Y. Anxiolytic effect of an extract of Salvia miltiorrhiza Bunge (Danshen) in mice. J. Ethnopharmacol. 2021, 264, 113285. [CrossRef]

2. Kang, H.S.; Chung, H.Y.; Jung, J.H.; Kang, S.S.; Choi, J.S. Antioxidant effect of Salvia miltiorrhiza. Arch. Pharmacal Res. 1997, 20, 496-500. [CrossRef]

3. Wang, X.; Gao, A.; Jiao, Y.; Zhao, Y.; Yang, X. Antitumor effect and molecular mechanism of antioxidant polysaccharides from Salvia miltiorrhiza Bunge in human colorectal carcinoma LoVo cells. Int. J. Biol. Macromol. 2018, 108, 625-634. [CrossRef]

4. Shi, M.; Huang, F.; Deng, C.; Wang, Y.; Kai, G. Bioactivities, biosynthesis and biotechnological production of phenolic acids in Salvia miltiorrhiza. Crit. Rev. Food Sci. Nutr. 2019, 59, 953-964. [CrossRef]

5. Li, Z.-M.; Xu, S.-W.; Liu, P.-Q. Salvia miltiorrhiza Burge (Danshen): A golden herbal medicine in cardiovascular therapeutics. Acta Pharmacol. Sin. 2018, 39, 802-824. [CrossRef] [PubMed]

6. Liu, H.; Wang, X.; Wang, D.; Zou, Z.; Liang, Z. Effect of drought stress on growth and accumulation of active constituents in Salvia miltiorrhiza Bunge. Ind. Crop. Prod. 2011, 33, 84-88. [CrossRef]

7. Lisar, S.Y.; Bakhshayeshan-Agdam, H.; Li, X.; Liu, F.; Burgess, P.; Huang, B.; Merewitz, E.; Zivcak, M.; Brestic, M.; Sytar, O.; et al. Drought Stress Tolerance in Plants; Springer: Berlin/Heidelberg, Germany, 2016; Volume 1. [CrossRef]

8. Zampieri, M.; Ceglar, A.; Dentener, F.; Toreti, A. Wheat yield loss attributable to heat waves, drought and water excess at the global, national and subnational scales. Environ. Res. Lett. 2017, 12, 064008. [CrossRef]

9. Chen, X. Effects of Drought Stress on Growth, Yield and Quality of Different Barley Genotypes. Master's Thesis, Zhejiang University, Hangzhou, Zhejiang, 2015.

10. Zhu, Z.; Liang, Z.; Han, R. Saikosaponin accumulation and antioxidative protection in drought-stressed Bupleurum chinense DC. plants. Environ. Exp. Bot. 2009, 66, 326-333. [CrossRef]

11. Jia, X.; Sun, C.; Li, G.; Li, G.; Chen, G. Effects of progressive drought stress on the physiology, antioxidative enzymes and secondary metabolites of Radix Astragali. Acta Physiol. Plant 2015, 37, 1-14. [CrossRef]

12. Xue, J.; Zhou, S.; Wang, W.; Huo, L.; Zhang, L.; Fang, X.; Yang, Z. Water availability effects on plant growth, seed yield, seed quality in Cassia obtusifolia L., a medicinal plant. Agric. Water Manag. 2018, 195, 104-113. [CrossRef]

13. Cao, M.; Liu, Y.; Jiang, W.; Meng, X.; Zhang, W.; Chen, W.; Peng, D.; Xing, S. UPLC/MS-based untargeted metabolomics reveals the changes of metabolites profile of Salvia miltiorrhiza bunge during Sweating processing. Sci. Rep. 2020, 10, 19524. [CrossRef]

14. Caser, M.; Chitarra, W.; D'Angiolillo, F.; Perrone, I.; Demasi, S.; Lovisolo, C.; Pistelli, L.; Pistelli, L.; Scariot, V. Drought stress adaptation modulates plant secondary metabolite production in Salvia dolomitica Codd. Ind. Crop. Prod. 2019, 129, 85-96. [CrossRef]

15. Xie, X.; He, Z.; Chen, N.; Tang, Z.; Wang, Q.; Cai, Y. The roles of environmental factors in regulation of oxidative stress in plant. BioMed Res. Int. 2019, 2019, 9732325. [CrossRef]

16. Yang, L.; Wen, K.-S.; Ruan, X.; Zhao, Y.-X.; Wei, F.; Wang, Q. Response of plant secondary metabolites to environmental factors. Molecules 2018, 23, 762. [CrossRef]

17. Younis, A.; Ramzan, F.; Ramzan, Y.; Zulfiqar, F.; Ahsan, M.; Lim, K.B. Molecular Markers Improve Abiotic Stress Tolerance in Crops: A Review. Plants 2020, 9, 1374. [CrossRef]

18. Chai, F.; Liu, W.; Xiang, Y.; Meng, X.; Sun, X.; Cheng, C.; Liu, G.; Duan, L.; Xin, H.; Li, S. Comparative metabolic profiling of Vitis amurensis and Vitis vinifera during cold acclimation. Hortic. Res. 2019, 6, 8. [CrossRef]

19. Villar, M.; Ayllón, N.; Alberdi, P.; Moreno, A.; Moreno, M.; Tobes, R.; Mateos-Hernández, L.; Weisheit, S.; Bell-Sakyi, L.; de la Fuente, J. Integrated metabolomics, transcriptomics and proteomics identifies metabolic pathways affected by Anaplasma phagocytophilum infection in tick cells. Mol. Cell. Proteom. 2015, 14, 3154-3172. [CrossRef] [PubMed]

20. Chen, Y.; Chen, Y.; Guo, Q.; Zhu, G.; Wang, C.; Liu, Z. Effects of drought stress on the growth, physiology and secondary metabolite production in pinellia ternata thunb. Pak. J. Bot 2021, 53, 833-840. [CrossRef]

21. Jang, S.-A.; Park, D.W.; Kwon, J.E.; Song, H.S.; Park, B.; Jeon, H.; Sohn, E.-H.; Koo, H.J.; Kang, S.C. Quinic acid inhibits vascular inflammation in TNF- $\alpha$-stimulated vascular smooth muscle cells. Biomed. Pharmacother. 2017, 96, 563-571. [CrossRef] [PubMed]

22. Muthamil, S.; Balasubramaniam, B.; Balamurugan, K.; Pandian, S.K. Synergistic effect of quinic acid derived from Syzygium cumini and undecanoic acid against Candida spp. biofilm and virulence. Front. Microbiol. 2018, 9, 2835. [CrossRef] [PubMed]

23. Heikkilä, E.; Hermant, A.; Thevenet, J.; Bermont, F.; Kulkarni, S.S.; Ratajczak, J.; Santo-Domingo, J.; Dioum, E.H.; Canto, C.; Barron, D. The plant product quinic acid activates $\mathrm{Ca}^{2+}$-dependent mitochondrial function and promotes insulin secretion from pancreatic beta cells. Br. J. Pharmacol. 2019, 176, 3250-3263.

24. Chu, S.; Liu, W.; Lu, Y.; Yan, M.; Guo, Y.; Chang, N.; Jiang, M.; Bai, G. Sinigrin Enhanced Antiasthmatic Effects of Beta Adrenergic Receptors Agonists by Regulating cAMP-Mediated Pathways. Front. Pharmacol. 2020, 11, 723. [CrossRef]

25. Lee, H.-W.; Lee, C.G.; Rhee, D.-K.; Um, S.H.; Pyo, S. Sinigrin inhibits production of inflammatory mediators by suppressing NFкB/MAPK pathways or NLRP3 inflammasome activation in macrophages. Int. Immunopharmacol. 2017, 45, 163-173. [CrossRef] [PubMed]

26. Jang, Y.J.; Park, B.; Lee, H.-W.; Park, H.J.; Koo, H.J.; Kim, B.O.; Sohn, E.-H.; Um, S.H.; Pyo, S. Sinigrin attenuates the progression of atherosclerosis in ApoE-/ - mice fed a high-cholesterol diet potentially by inhibiting VCAM-1 expression. Chem. Biol. Interact. 2017, 272, 28-36. [CrossRef] [PubMed] 
27. Ommati, M.M.; Heidari, R.; Ghanbarinejad, V.; Abdoli, N.; Niknahad, H. Taurine treatment provides neuroprotection in a mouse model of manganism. Biol. Trace Elem. Res. 2019, 190, 384-395. [CrossRef]

28. Guo, J.; Ma, Y. Biosynthetic Pathway of Tanshinones in Salvia miltiorrhiza. In The Salvia miltiorrhiza Genome; Springer: Berlin/Heidelberg, Germany, 2019; pp. 129-139.

29. Fu, L.; Han, B.; Zhou, Y.; Ren, J.; Cao, W.; Patel, G.; Kai, G.; Zhang, J. The anticancer properties of tanshinones and the pharmacological effects of their active ingredients. Front. Pharmacol. 2020, 11, 193. [CrossRef]

30. Liu, Z.; Zhu, W.; Kong, X.; Chen, X.; Sun, X.; Zhang, W.; Zhang, R. Tanshinone IIA inhibits glucose metabolism leading to apoptosis in cervical cancer. Oncol. Rep. 2019, 42, 1893-1903. [CrossRef]

31. Qin, T.; Rasul, A.; Sarfraz, A.; Sarfraz, I.; Hussain, G.; Anwar, H.; Riaz, A.; Liu, S.; Wei, W.; Li, J. Salvianolic acid A \& B: Potential cytotoxic polyphenols in battle against cancer via targeting multiple signaling pathways. Int. J. Biol. Sci. 2019, 15, 2256.

32. Haberstroh, S.; Kreuzwieser, J.; Lobo-do-Vale, R.; Caldeira, M.C.; Dubbert, M.; Werner, C. Terpenoid emissions of two Mediterranean woody species in response to drought stress. Front. Plant Sci. 2018, 9, 1071. [CrossRef] [PubMed]

33. Kleiber, A.; Duan, Q.; Jansen, K.; Verena Junker, L.; Kammerer, B.; Rennenberg, H.; Ensminger, I.; Gessler, A.; Kreuzwieser, J. Drought effects on root and needle terpenoid content of a coastal and an interior Douglas fir provenance. Tree Physiol. 2017, 37, 1648-1658. [CrossRef] 